This article was published as: Journal of Nanofluids 5 (4), 479-495, 2016

DOI:10.1166/jon.2016.1233 


\title{
Mechanics of Magnetopolymer Composites: a Review
}

\author{
M. T. Lopez-Lopez ${ }^{1}$, Juan D. G. Durán, ${ }^{1}$ L.Yu.Iskakova ${ }^{2}$, A.Yu.Zubarev ${ }^{2}$ \\ ${ }^{1}$ Departamento de Física Aplicada, Facultad de Ciencias, Universidad de Granada, Campus de \\ Fuentenueva, 18071 Granada, Spain,
}

${ }^{2}$ Urals Federal University, Lenina Ave 51, 620083, Ekaterinburg, Russia.

\begin{abstract}
Magnetopolymer materials (ferrogels, magnetic hydrogels, magnetic elastomers) are new kind of smart materials whose physical and mechanical properties can be controlled by an external magnetic field. We present an overview of experimental, theoretical and computer studies of magentomechanical properties and behavior of these systems.
\end{abstract}

KEYWORDS: Ferrogels, Magentostriction, Elastic moduli.

\section{INTRODUCTION.}

The possibility to control physical properties and behavior of polymer and other soft matters by using magnetic fields is very attractive for many industrial and bio-medical applications. The main problem here is in the fact that all known natural soft matters are diamagnetic. Therefore, control of their properties requires very strong magnetic fields and bulky apparatuses. The effective solution of this problem gives the composites of fine (nano- and micron-sized) magnetic particles in polymer matrixes. Coupling of high elasticity and reaction on magnetic field; strong magnetomechanic effects; sharp (several orders of magnitudes) dependence of electroconductivity on pressure and external magnetic field; dependence of mechanical modulus on applied field as well as other unique properties of these systems attract considerable interest of researches and engineers.

The usual way of the composites manufacturing is in incorporation of a magnetic filler particles in liquid solution or melt of a polymer and curing the matrix by using special chemical agents. If magnetic field is applied to the systems during the cross-linking, magnetic particles can form various anisotropic structures (chains, dense columns, etc). The resulting polymerized composite is macroscopically anisotropic; very often macroscopical properties of the anisotropic systems significantly differ from those of the similar systems cured without magnetic field. 
One of the attractive, from the viewpoint of applications, and challenging, for the scientific study, features of magnetic polymers is dependence of their internal architecture and macroscopical properties on external magnetic field. It opens the possibility to control their behavior with the help of quite moderate magnetic field in the range of $10-100 \mathrm{kA} / \mathrm{m}$. In part, these composites are used as magnetocontrollable dampers, vibration and shock absorbers [1-6]; temperature, pressure and chemical sensors [7-12]. In electronics they are used as protections against electromagnetic radiation and electrostatic discharge [13], as electrical conductive adhesive for electrical connections [14]. Hydrogels with biocompatible magnetic particles are very promising for biomedical applications - for drug delivery and biosensors $[15,16]$, biopolymeric scalfolds [17-35].

Tissue engineering is an interdisciplinary field that applies the principles of biology and engineering toward the development of strategies aimed at the replacement, repair, maintenance, and/or enhancement of tissues [36]. Novel techniques for tissue repair are based on the use of biodegradable polymeric scaffolds, which are grafted in the lesion site, in combination with cells and proteins to promote cell adhesion and proliferation [37]. The role of the scaffold in tissue engineering is determinant and its use is constantly increasing.

Recently several authors proposed the use of magnetic nanoparticles in combination with polymers for the preparation of innovative magnetic scaffolds (ferroscafolds or ferrogels) for tissue substitutes [16-23, 25-31,38,39].

Several advantages are mentioned for these ferrogels. Firstly, the magnetic behavior of the ferrogels will allow visualization by magnetic resonance [31]. Secondly, in-vitro studies indicate that the presence of the magnetic nanoparticles in the scaffolds stimulate adhesion, proliferation, and differentiation of cells [27]. In addition to these, the main advantage of novel magnetic biocompatible ferroscaffolds seems to be their magnetic moment under an external magnetic field, which will make them work as magnets, attracting functionalized magnetic nanoparticles injected close to them $[16,31,40]$. This represents a promising strategy to guide and accumulate in the scaffolds growth factors, drug doses and cells attached to the injected magnetic nanoparticles.

In addition, very recently the possible to reversibly tune the mechanical properties of biocompatible ferroscaffolds by application of external magnetic fields has been demonstrated in [33]. This is an unique advantage with respect to other scaffolds that could be used to match the mechanical properties of the tissue substitutes to those of potential target tissues in tissue engineering applications [33]. 
Depending on the size of the particles and mechanical properties of the matrix, magnetic polymers are named as ferrogels [41, 42], magnetoelastics [43,44], elastomer-ferromagnetic composites [45], soft magnetic elastomers [46]. The difference between magnetic gels and elastomers is in mechanical properties of the polymer matrix - the first ones are much softer than the second ones. Very often the systems of micron-sized magnetizable particles in elastomer matrix are termed as magnetic either magnetorheological elastomers [47].

In this review we focus on magnetomechanical effects in magnetic polymers- behavior and properties of systems under the action of external magnetic field

\section{MAGNETOSTRICTION.}

One of the interesting and challenging problems of soft ferrogels behavior is their ability for elongation or contraction under the action of applied magnetic field (magnetostriction effect). Simple estimates (see, for example, [44]) show that linear deformation of soft magnetic gels, under the action of uniform magnetic field of the strength about $100 \mathrm{kA} / \mathrm{m}$, can achieve several tens per cent. That is several orders of magnitudes more than magnetostriction in any known solid magnets. Possibly, for the first time the magnetostriction effects (elongation and contraction of ferrogels under the action of magnetic field) have been observed and studied in the works $[48,49]$. The composites have been placed in non-uniform field. The magnetite particles with the mean diameter $10 \mathrm{~nm}$ were incorporated in the chemically cross-linked gels. It was shown that concentration of magnetic particles and the cross-linking density of the ferrogels play an important role in the magneto-mechanical effects. Influence of magnetic field on the elastic properties of the composites has not been detected. The last result can be explained by the small size of the particles and, as a consequence, weak magnetic interaction between them.

Dependences of absolute values of the sample deformations as functions of the electrical current in electromagnets, created the non uniform field, have been measured in these works. Unfortunately, the relative deformations as well as magnitudes of gradient of the field have not been presented. It was noted, that after the switching on (off) the non uniform magnetic field, the same deformation occurred very fast - for hundredth of second. Unfortunately, dependence of size and shape of the samples on their deformation has not been detected. One needs to note that independence of the kinetics on the sample size looks quite natural; this independence on the sample shape is rather a consequence of the uniformity of the field.

These investigations have been continued in [50,51] where a discontinuous elongation and contraction in response to infinite-small change in the external magnetic field as well as hysteresis's of the sample deformation vs. the current in electromagnets have been observed. A theoretical interpretation of these effects has been suggested. From the physical point of view, 
the discontinuity of the deformations and the hysteresis effects were explained by the non uniform space distribution of magnetic field between the poles of the electromagnets and the rearrangement of the particles in the sample under the field action.

Various experiments show that the type (contraction or elongation) and value of the magnetostriction significantly depend on the space arrangement (either random isotropic or anisotropic with heterogeneous aggregates), concentration and size of the particles, shape of the sample and many other factors. As, the magnetostrictions of ferrogels with randomly distributed particles have been studied in [52]. The explored composites were cylindrically shaped and consisted of the moderately soft gel matrix, with the Young's modulus about $100 \mathrm{kPa}$, filled by iron particles of $5 \mu \mathrm{m}$ in diameter. Volume concentration of the particles in the composites was about thirty per cent. The results of measurements of the magnetostriction versus the applied magnetic field, of samples with different aspect ratios are presented. Only elongation of the samples under the action of the uniform field has been detected, hysteresis dependences of the elongation on the field were observed. The experiments displayed shape dependence of the magnetodeformation - elongation of the flat samples was more than that of the prolate ones.

The measurements showed that the Young's modulus $E$ of the composites monotonic, parabolic-like, increased with concentration of the particles. It was noted that for relatively small volume concentrations $\varphi$ of the particles $(\varphi<15-20 \%)$ this dependence well corresponds to the Guth and Gold law

$E=E_{0}\left(1+2.5 \varphi+14.1 \varphi^{2}\right)$

where $E_{0}$ is the modulus of the pure gel matrix. For larger concentrations this law underestimates the experimental results. In the whole studied region of concentrations $(0<\varphi<35 \%)$ the Maron-Pierce folrmula

$E=E_{0}\left(1-\frac{\varphi}{\varphi^{*}}\right)^{-2}$

allows to achieve reasonable agreement with experiments. Here $\varphi^{*}$ is defined as maximum of packing fraction. In [52] it was estimated as $\varphi=54 \%$.

The experimentally determined dependence of the elongation $\varepsilon$ on the concentration $\varphi$ has a maximum at $\varphi \approx 25-30 \%$. Analysis shows that this maximum is a result of competition between increase, with $\varphi$, of the elongating magnetic forces, acting on the sample on the one hand, and increase of the Young's modulus $E$, on the other hand. The last factor, obviously, leads to reduction of the elongation.

A theoretical model based on the estimate of the total energy $U$ of the sample deformation is suggested in [52]. The energy $U$ consists of two terms. The first one $U_{\mathrm{el}}$ is the term of elastic 
deformation; for small $\varepsilon$ it is proportional to $E \varepsilon^{2}$. The second one corresponds to the change of the magnetic part $U m$ of the sample energy. This term includes the demagnetizing shape factor $N$, which decreases while the sample elongation. According to [52], the change of the magnetic energy $U_{m}$ stimulates elongation of the sample in the field direction, whereas the elastic effects restrict this deformation. The equilibrium elongation can be found from the relation $\partial U / \partial \varepsilon=0$. One needs to note that the explicit analytical forms for the demagnetizing factor $N$ are known only for ellipsoidal samples (see, for example, [53]), whereas in the experiments [52] the samples had the cylindrical shape. Unfortunately the mathematical forms of $N$, used in the calculations, are not presented in [52]. It is interesting to note that the experimentally detected non monotonic dependence of $\varepsilon$ on $\varphi$ has been reproduced theoretically only by using the estimate (2) for the Young's modulus, whereas the formula (1) leads to monotonic increase of $\varepsilon$ on $\varphi$.

It is important that in reality the magnetic energy $U_{m}$ changes after the sample deformation not only because of change of the demagnetizing shape factor $N$, but also due to the change of effective susceptibility $\chi$ of the composite [53]. The change of the susceptibility takes place due to the change of the mutual disposition of the particles in the sample after its deformation. This factor has not been taken into account in [52].

Magnetostriction effects in elastomers with micron-sized magnetic particles united in chain-like aggregates have been studied in [47]. The chains were formed by application of external magnetic field on the stage preceding the matrix curing. The prepared samples consisted of the matrix with the elastic modulus $230 \mathrm{kPa}$ filled by the iron particles with $10 \%$ of the volume concentration. Unlike magnetoelongation of the samples with the random isotropic distribution of the particles, observed in [52], experiments [47] demonstrated contraction of the samples in the field direction due to shrink of thin gaps between the particles in the chains. By the action of the field $120 \mathrm{kA} / \mathrm{m}$ the detected contraction was about $1.4 \%$ of the initial length. Simple models, based on the concepts of perfect chains and the chains with defects, have been suggested to explain the observed magnetostriction. Theoretical results are in reasonable agreement with the experiments. Besides the mechanical effects, influence of the elastomer deformations on electroresistivity of the composites has been studied in [47]. To this end the samples with the nickel particles of the $30 \%$ of the volume concentration were used. Like in the samples with the iron particles, the nickel ones were united in the anisotropic chain-like structures due to magnetic field applied to the synthesized composite. Experiments demonstrated that the unstrained sample was practically perfect isolator with the resistivity about $10^{6} \Omega \mathrm{m}$; the sample deformation with the strain $40 \%$ transformed the composite to good conductive material with the resistivity about 
$2 \Omega \mathrm{m}$. This strong piezzoresistivity has been explained by reorganization of the particles in the chains, due to this deformation, and by appearance direct conductivity between the particles in the percolating chains.

Piezziresistivity of magnetic elastomers theoretically and experimentally has been studied also in [54]. Experiments of this work have shown that the resistivity of the composites with anisotropic internal structures is significantly less that the resistivity of the isotropic composites. Theoretical model of the tunnel conductivity through the contact spots between the particles is developed. The model allows quantitatively describe the experimental results.

Thermo-and magneticresistance effects in the elastomers with the structures have been explored in [55]. The experimental results demonstrated giant (seven orders of magnitude) increase of the sample resistance while the temperature increased from 20 to $50{ }^{\circ} \mathrm{C}$. External magnetic field, in the range from 0 to $200 \mathrm{kA} / \mathrm{m}$, induced strong decrease of the resistance - more than order of magnitude with the composites containing $10 \%$ of volume concentration of the filler and about four orders of magnitudes for the samples with $30 \%$ of the filler. These experimental results are explained by the tunnel conductivity between the particles. As, magnetic interaction of the magnetizable particles reduces the ultrathin gaps between them. This enhances the tunnel conductivity. The theoretical results well reproduce the experimental ones. The results of $[47,54,55]$ show that the magnetic elastomers can be used for making of sensors of external pressure, temperature and magnetic field.

\section{MAGNETOMECHANICAL PROPERTIES AN BEHAVIOR.}

Experimentally effect of magnetic field on the mechanical properties of magnetic polymers has been studied in [56]. The composites consisted of the carbonyl iron particles embedded in a silicon elastomer; the mean diameter of the particle was $2 \mu \mathrm{m}$. Two kinds of the samples have been prepared. The first ones are with homogeneous and isotropic spatial distribution of the particles. These composites have been cured without external magnetic field. The samples of the second type were polymerized under the action of the field; the particles, in these composites, formed long chain-like aggregates aligned along the field. The dependence of the elastic stress versus elongation strain has been detected for the samples with the particles volume fractions ranged between 5 and 25 per cent. External magnetic field were applied along the line of deformation. The samples with the anisotropic structures have been deformed in the direction of the anisotropy axis. The measurements have been performed in the region of the strain $\varepsilon$ deformation up to 10 per cent of the initial length of the samples. In this range of deformation, the linear relation between the stress and deformation has been detected for the pure silicon matrix. 
The experiments demonstrated very weak, barely visible, effect of magnetic field $H$ on the elastic stress $\sigma$ in the isotropic composites. In contrast, in the systems with the anisotropic structures effect of the field on the stress was quite pronounced and achieved several tens per cent. In these systems, placed in magnetic field, an initial stress, corresponding to zero deformation, has been observed; the magnitude of the stress increased with the applied field. In the anisotropic samples the stress non-linearly depended on the relative elongation $\varepsilon$, whereas in the isotropic systems this relation was linear, like in the pure matrix.

Non-monotonic, $N$-shaped dependence of the stress difference $\Delta \sigma=\sigma(H)-\sigma(0)$ on the strain $\varepsilon$ has been observed in [56] (Fig.1 b). Authors have explained this dependence by the presence, in the composite, of the chains with different length. According to their modeling, the chains of the maximal length can be ruptured at the relatively small strain. This rupture must lead to abrupt decrease of the macroscopical stress whereas the elastic resistance of the shorter chain stimulates the monotonic increase of the stress. In this concept the combination of the effect of the chains with the difference length leads to smooth non monotonic dependence of $\Delta \sigma$ on $\varepsilon$.

The measurements of the storage $E^{\prime}$ and loss $E^{\prime \prime}$ parts of the complex Young's modulus at the oscillating deformations have been carried out at the frequency of the oscillations $5 \mathrm{~Hz}$. The experiments have demonstrated monotonic decrease of $E^{\prime}$ and non monotonic, with maximum, dependence of $E^{\prime \prime}$ on the strain amplitude.

Non linear relations between the elastic stress and strain in magnetic elastomers with internal structures has also been observed in [42]. Volume concentration of magnetic filler in these experiments was in the range 30-37 per cent. Without magnetic field practically the linear dependence of the stress $\sigma$ on the strain $\varepsilon$ has been observed for all samples. In experiments with magnetic field, applied along the line of the elongation, the non linear dependence of $\sigma$ on $\varepsilon$ has been detected - for small (1-5\%) elongations the stress increased much faster with the strain than at higher elongations. To characterize the non-linear elasticity of the sample the differential Young's modulus $E=\mathrm{d} \sigma / \mathrm{d} \varepsilon$ has been used. The experiments showed that for the field about $250 \mathrm{mT}$ this modulus decreased about three orders of magnitudes when the strain $\varepsilon$ varied in the range from 0 to $35 \%$. Significant, about 7-8 times, increase of the initial (corresponding to zero deformation) shear modulus under the action of the field $80 \mathrm{mT}$ has been detected. Increase of the shear strain leads to fast decrease of the shear modulus. Without magnetic field the modulus practically does not depend on the magnitude of the shear. In magnetic field the stress - strain curves demonstrated hysteresis behavior.

The shear storage $G^{\prime}$ and loss $G^{\prime \prime}$ modulus, corresponding to the oscillating deformations of the samples, has been measured as functions on the oscillations frequency and applied magnetic 
field. First, the experiments demonstrated significant increase both $G^{\prime}$ and $G^{\prime \prime}$ under the field action. This effect has been explained by the arrangement of the particles after the field application. In magnetic field the storage modulus monotonic increased with the frequency in the explored range from 0 to $5 \mathrm{~Hz}$; the loss modulus - decreased.

If the polymer matrix is soft enough and magnetic interaction between the particles is strong, one can expect significant rearrangement of the particles in the composites and formation of various heterogeneous structures. This rearrangement can lead to strong change of macroscopical properties and behavior of the composites. In experiments [57] formation, under the action of applied magnetic field, of the chain-like structures in ferrogels with initially chaotic and isotropic spatial distribution of the particles, has been observed. The carbonyl iron particles with diameter 2-4 $\mu \mathrm{m}$ embedded in silicon matrix have been used in these experiments; volume fraction of the magnetic phase was $30-35 \%$.

Measurements of the mechanical properties of the composites demonstrated that, as in experiments [42], under magnetic field the stress-strain dependences formed wide hysteresis loops, whereas without magnetic field no hystereses were observed. The sample magnetized under some magnetic field, stretched and then unloaded, kept the acquired strain during the whole time of observation (hours). Decrease of applied magnetic field led to reduction of the residual strain.

Elongation of the samples in the direction of applied magnetic field has been observed. Dependence of the strain on the field, like in [52], had the hysteresis character. The sample magnetization, as function of the applied magnetic field, also demonstrated the hysteresis behavior. The physical cause of the hysteresis effects is in formation, under the field action, of the chain-like structures. Analysis shows that the length of the chains experiences the hysteresis dependence on the applied magnetic field and on the macroscopical strain. This leads to the macroscopic hysteresis effects.

The measurements have shown that without external magnetic field the sample Young's modulus does not depend on the strain in the range of deformation till $10 \%$ of the length. In strong magnetic field this modulus non monotonic, with sharp maximum, depends on the strain. The microscopic cause of this effect is unclear.

The performed experiments demonstrated non monotonic, with maximum, dependence of the sample magnetic susceptibility on applied magnetic field. In relatively soft composites the observed susceptibility was more and the maximum was more pronounced than in the relatively hard systems. These results also can be explained by the chaining of the particles under the field action. Unification of the particles in the chains leads to initial increase of the susceptibility since demagnetizing factor of the chain is less than that of the single particle. The further reduction of 
the susceptibility with the field is explained by the usual decreasing dependence of the particle material susceptibility on the field.

Another problem of the magnetomechanical behavior of the composites has been studied in [58]. In these experiments the static elastic Young's modulus of the relatively rigid magnetic elastomers with the micron-sized iron particles were measured. Two types of the samples have been explored: with the randomly distributed particles and with the particles united in chain-like aggregates as well. The weight concentrations of the magnetic filler in these samples were 10, 20 and 30 per cent. The main goal of this work was to study effect of the relative orientations of the chains, applied magnetic field and external force on the elastic modulus of the composites.

Experiments have shown that increase of magnetic field from 0 to $400 \mathrm{mT}$ induced increase of the elastic modulus of the isotropic samples in the range about 10 per cent. Effect of the field direction, with respect to the mechanical force, on the elastic modulus was quite weak. In contrast, the magnetoelastic effects in the systems with the anisotropic structures appeared very sensitive to the directions of the field and the force. The maximal increase of the modulus under magnetic field action (about two times for the composite with $30 \%$ wt of the filler) has been detected when both the field $\mathbf{H}$ and the mechanic force $\mathbf{F}$ were aligned along the internal chains. The weakest effect was observed when both the $\mathbf{H}$ and $\mathbf{F}$ were perpendicular to the chains.

Mechanical properties a polyurethane foil-shaped elastomers with various volume concentrations $(20 \% ; 40 \% ; 60 \%)$ of magnetic particles have been studied in [59]. The elastic moduli, corresponding to deformation in three directions with respect to the applied field, were measured in dependence on the field. The modulus increases with the field for all concentrations of the particles. Magnetostriction (contraction in the field direction) has been observed. For the samples with the concentration 40 and $60 \%$, the magnitude of the striction monotonic increases with the field. Surprisingly, the striction for the composite with $20 \%$ of the particle concentration non monotonic (with the maximum of the absolute value) depends on the field.

Effect of internal structures on rheological properties of magnetic elastomers have been studied also in [60]. In this work the large-strain behavior of magneto-rheological elastomers (MREs) is characterized experimentally under uniaxial compression, uniaxial tension and pure shear deformation, in the absence and in the presence of magnetic fields. Silicone matrix was filled by magnetizable carbonyl iron particles of the size about $4 \mu \mathrm{m}$. The experiments have been done with the isotropic and anisotropic composites. In all cases the systems with the anisotropic internal structures were more rigid than the isotropic ones. Magnetic field practically does not affect elastic properties of the isotropic samples, but significantly increases those for the anisotropic systems. 
Like in [57], the experiments [60] demonstrate the hysteresis loop for the dependence of stress versus strain; the elastic moduli increase with the sample deformation.

The samples with randomly dispersed and aligned carbonyl iron particles were studied in [2] Scanning electron and light microscopy techniques were used for the MRE microstructure observations. The rheological properties (storage and loss modulus) of the MRE were tested without and with the application of the magnetic field. It was found that the microstructure of MREs, particularly the amount and arrangement of the carbonyl-iron particles, has a significant influence on their rheological and damping properties. Magnetic field increases the modulus of the system with internal structures.

Effect of polymer matrix on the magnetomechanical phenomena have been investigated in [61]. To these end different MR elastomers, one made of polyurethane and the other made of natural rubber, were synthesized. The composites were cured in magnetic field to create internal anisotropic heterogeneous structures. The experimental results show that the elastic shear modulus of polyurethane MR elastomers can increase by $28 \%$ under a strong magnetic field. Comparatively, the rubber MR elastomer has low modulus change ability. For the both composites the modulus decreases with the shear strain. A mathematical model to represent the stress-strain relationship of the MR elastomers is presented. The model takes into account the dipole-dipole interactions between the particles in a chain and the nonlinear properties of the host composite. The analytical results of the model are in agreement with experimental data.

In [62] an experimental investigations of magnetorheological gels under dynamic and static shear conditions in the presence of a magnetic field were performed. MR gels were prepared with micron sized polarizable carbonyl iron particles interspersed in a polymer matrix gel. The compliance of this magnetic gel can be varied under the influence of an external magnetic field. Since dynamical mechanical analysis tests are difficult to conduct in the presence of large deformations of the order of $50 \%$ and strong magnetic fields, a free decay test apparatus is designed and fabricated for obtaining the magnetic field dependent shearing response under dynamic conditions at room temperature.

It was observed that a significant change in the elastic modulus occurs in the gels under a magnetic field in the range of $0.1-0.4 \mathrm{~T}$. However, no significant change in the damping ratio was detected under various magnitudes of magnetic field. It was shown that the increase in shear modules of this kind of magnetic composite gel could be as high as $59 \%$ of the zero field value for a gel prepared with $50 \%$ by weight of carbonyl iron particles.

Combined experimental and theoretical study of the macroscopic response of MRE consisting of a rubber matrix phase with spherical carbonyl iron particles is presented in [63]. The MRE specimens used in this work are cured in the presence of strong magnetic fields leading to the 
formation of particle chain structures and thus to an overall transversely isotropic composite. The MRE samples are tested experimentally under uniaxial stresses as well as under simple shear in the absence or in the presence of magnetic fields and for different initial orientations of their particle chains with respect to the mechanical and magnetic loading direction. A transversely isotropic energy density function is proposed to reproduce the experimentally measured magnetization, magnetostriction and simple shear curves under different prestresses, initial particle chain orientations and magnetic fields. Microscopic mechanisms are also proposed to explain $(i)$ the counterintuitive effect of dilation under zero or compressive applied mechanical loads for the magnetostriction experiments, and (ii) the importance of a finite strain constitutive formulation even at small magnetostrictive strains. The microscopic analysis takes into account that the chains can have not the perfectly straight structure, but rather fluctuating shape. Experiments and the model demonstrate that, depending on the chain shape, magnetic field can either elongate or contract the sample in the field direction. The model gives an excellent agreement with experiments for relatively moderate magnetic fields but has also been satisfactorily extended to include magnetic fields near saturation.

Effect of shape of the particles of the magnetic filler on macroscopical properties of the composites presents significant interest because varying the shape one can achieve wide variation of these properties. As, the shear modulus of hydrogel with $N i$ nanorods have been studied in [64]. Magnetization measurements of the ferrogels show a significant influence of the gelatine concentration on their magnetic behavior. In particular, with decreasing gelatine concentration the measured coercivity is reduced indicating a mechanical rotation of the nanorods in the field direction. A theoretical model which relates the measured coercivity to the shear modulus of the surrounding gel matrix is introduced and applied to investigate the ageing process of gelatine gels with different gelatine concentrations at room temperature. Experiments demonstrate increasing dependence of the composite shear modulus and magnetic coercetivity on the time of the composite gelation. Unfortunately, no comparisons of the measurements with respect to the ones for the composites of the spherical particles have been presented. That is why it is difficult to make a conclusion on effect of the particle shape on the studied phenomena.

The discussed results of [56-63] show that macroscopical properties of magnetic composites strongly depend on the peculiarities of the particles spatial disposition in the materials. One can expect that deformation of the sample can lead to some rearrangement of the particles; in its turn this rearrangement can lead to significant change of physical and mechanical properties of the systems. As, the giant decrease of the storage modulus $G^{\prime}$ of a ferrogels with the micron-sized particles after small (about $10^{-3}$ ) relative elongation, due to the magnetostriction, of the samples 
have been detected in experiments [65]. Absolute value of the modulus reduction increased with volume concentration $\varphi$ of the magentic filler.

Experimental study of dependence of the storage and loss modulus on the elongation strain, before the magentostriction, have been performed. Significant, about 2 times, reduction of the modulus, in the range of the elongation between $10^{-6}$ and $10^{-2}$, has been observed. The experiments have been performed for several volume concentrations of the particles, from 0 to $24 \%$. Initial, for zero starin, value of $G^{\prime}$ fast increases with the concentration. In contrast, for elongation of order $10^{-2}$ this modulus is practically indepent on $\varphi$ and is very close to the modulus of the pure polymer matrix.

For all explored samples the loss tangent exhibited significant increase with the magnitude of the strain. Authors explain this effect by the enhanced nonlinear viscoelasticity resulting from the mechanical loss owing to the destruction of the particles network.

The experiments have been performed with the composiets containing the ferrite-barium and with the iron oxide particles as well. In should be noted that all magnetomechanical effects in the ferrite-barium systems were significantly stronger than those in the iron oxide ones.

The magnetoelastic behavior of bimodal magnetic elastomers consisting of magnetic, carbonyl iron particles and nonmagnetic, zinc oxide particles have been investigated by dynamic viscoelastic measurements in [66], [67]. One needs to note that the hybrid composites, consisting of the magnetic and non magnetic particles, present interest because they demonstrate significant magnetomechanical effects; on the other hand they can be much cheaper than the systems with the similar properties, but containing only of magnetic particles.

The experiments have demonstrated increase of the storage modulus of the magnetic elastomers under a magnetic field of $500 \mathrm{mT}$. The change in the storage modulus was enhanced by adding nonmagnetic particles at volume fractions above a certain volume fraction of 0.02 , indicating the occurrence of stress transfer by a chain structure of magnetic particles via nonmagnetic particles. The critical volume fraction at $500 \mathrm{mT}$ determined by percolation analysis was nearly independent on the diameter of nonmagnetic particles. However, at low magnetic fields below $160 \mathrm{mT}$, the critical volume fraction was found to decrease with the particle diameter. Substituting magnetic particles with nonmagnetic ones, the change in the storage modulus of bimodal magnetic elastomers monotonically decreased with the substitution ratio of magnetic particles. According to the experiments of particle substitution, the storage modulus monotonically decreased with the substitution ratio of magnetic particles, however, it was obviously higher than those of monomodal magnetic elastomers It was concluded that the enhancement of magnetoelastic response for bimodal magnetic elastomers is intrinsically due to 
the increment in the probability of contact between nonmagnetic particles and the chains of magnetic particles. Elastic module increased with magnetic field.

A magnetorheological elastomers of new type consisting of polyurethane and carbonyl iron particles were obtained in [68]. These composites demonstrated drastic and reversible changes in dynamic modulus in air. The magnetic elastomer with a volume fraction of 0.29 exhibited a reversible increase by factors of 277 of the storage modulus and 96 of the loss modulus upon a magnetic field of $500 \mathrm{mT}$. The elastomer underwent high mechanical toughness with a braking strain exceeding 0.8 , and demonstrated the giant magnetorheological behavior for half a year after the synthesis without degradation.

It was noted in Introduction, that magnetic hydrogels are perspective material for medical and biological applications. Physically cross-linked isotropic and anisotropic poly(vinyl alcohol) (PVA) hydrogels containing micron-sized carbonyl iron particles were synthesized and studied in [69]. The materials were prepared through a cyclic freezing-thawing process. The PVA hydrogel can respond to a magnetic field and shows a magnetorheological (MR) effect, i.e., the modulus of the PVA hydrogel can be adjusted under a magnetic field. The chain-like structures of carbonyl iron are formed in the PVA hydrogel after orientation under a magnetic field of 1.5 T. Also some magnetic field induced oriented pores with a tunable diameter are observed in the dried PVA gel. Experiments have demonstrated that the MR effect can be adjusted by changing the carbonyl iron content, the initial concentration of PVA solution and test frequency. The formation of aligned chain-like structures of carbonyl iron in the anisotropic PVA MR hydrogel improves the compression properties and the MR effect. At a carbonyl iron content of $70 \mathrm{wt} \%$, the maximum absolute and relative MR effect of anisotropic PVA MR hydrogels are $1.24 \mathrm{MPa}$ and 230\%, respectively. The PVA hydrogels with good MR effects and moderate mechanical strength have potential applications in artificial muscle, soft actuators and drug release.

Magnetic and mechanical properties of hydrogels, fabricated from a fibrin matrix and biocompatative magnetic particles with diameter about 100nm have been studied in [33]. External magnetic field was applied at the start of the polymer gelation. After the composite curing parallel columns consisting of closely packed polymer fibers, separated by more isotropic net-like spaces, have been observed. Apparently, the columns appeared due to tendency of the particles, adsorbed on the fibers, to the phase condensation. The domains, with the relatively high concentration of the particles, elongates in the field direction.

The elastic shear moduli were measured at the oscillating strain with the frequency $1 \mathrm{~Hz}$. The measurements have demonstrated fast (much faster than the classical theories of composite materials [70] predict) increase of the shear modulus with concentration of the particles and fast decrease of these modulus with the shear strain. Possibly, the physical cause of the strong 
deviation of the experimental results from the theoretical ones is in the ratio between the size $a$ of the particles and the typical size $L$ of the cells of the polymer net. In the hydrogels, used in [33], the strong inequality $a<<L$ is held, whereas in the classical theories the opposite inequality is supposed; the matrix, with respect to the particles, is considered as a continuous medium.

Very interesting dependence of magnetic elastomer shear modulus on concentration of the magnetic filler have been observed in experiments [71]. The composites consisted of micronsized iron particles embedded in a silicon matrix. The cylindrical-shaped samples were polymerized under the action of magnetic field parallel to its axis if symmetry. In the samples with the particles concentration $\phi=5 \mathrm{wt} \%$, column-like structures, uniformly distributed in the volume, were observed. In the samples with the weight concentration $\phi$ more than $25 \%$, densely packed structured appeared.

Unexpectedly, the decreasing part of $G$ ' versus the weight concentration has been detected. The physical cause of this decreasing dependence is unclear.

\section{THEORETICAL STUDY AND COMPUTER SIMULATIONS}

One of the first theoretical studies of the mechanical properties of magnetic composites, based on the microscopical approach, has been done in [72]. The shear moduli of magnetic elastomer with magnetizable particles united in linear chains were calculated. Elastomer treated as hyperelastic system by using the Ogden-potential representation for the energy of elastic deformations

$U_{e l}=\sum_{i=1}^{3} \frac{2 \mu_{i}}{\alpha_{i}^{2}}\left(\lambda_{1}^{\alpha_{i}}+\lambda_{2}^{\alpha_{i}}+\lambda_{3}^{\alpha_{i}}-3\right)$

Here $\alpha_{i}$ и $\mu_{i}$ are adjusted parameters, $\lambda_{k}$ are the ratio of the actual sample length to the principal length in the $k$-direction. It was supposed that the composite was cured under magnetic field and the particles formed long chains with thin gaps between the particles.

The stress-strain relationship for MR elastomers has been calculated numerically, by using the structural finite element analysis. The result demonstrated that reduction of the gap between particles in the chain leaded to modest increase of the composite shear modulus $G$. Unexpectedly it was obtained that without magnetic field, even for the smallest gap considered, the calculated $G$ for the elastomer with aligned chains was less than that for the same elastomer with randomly dispersed particles.

In the limiting case of the infinitely strong magnetic field $\mathrm{H}$ the shear modulus has been estimated analytically by using the approximation of the dipole-dipole interaction between the 
particles. The change $\Delta G$ of the modulus in high-field limit with respect to that without field was estimated as:

$\Delta G=4.8 \mu_{0} \varphi M_{s}^{2}\left(\frac{2 a}{l}\right)^{3}$

where $\mu_{0}$ is the vacuum permeability, $M_{s}$ is saturated magnetization of the particle, $a$ is the particle radius, $l$ is the distance between the nearest particles centers in the chain. Calculations showed that, at the field of saturation, the magentoelastic effect, i.e. the ratio $(G(H)-G(0)) / G(0)$, was about $50 \%$; this ratio non monotonic depended on the particle volume concentration $\varphi$ and achieved maximum at the concentration $27 \%$. Unfortunately the direction of the imposed deformation with respect to the internal chains was not discussed.

A semi-empirical model of effect of chain-like aggregates on the shear modulus of magnetic elastomers is suggested in [73]. The particles are aligned in the perfect chains with gaps between the particles. All chains have the same length. Magnetic interaction only between nearest particles in the chain has been taken into account by using the dipole-dipole approximations. In the framework of this approximation, the energy, per the composite unite volume, of magnetic interaction between the particles in the sheared sample in [73] is determined as:

$U_{m}=\frac{3 \varphi\left(\epsilon^{2}-2\right) m^{2}}{2 \pi^{2} \mu_{0} \mu_{1} d^{3} l_{0}^{3}\left(1+\epsilon^{2}\right)^{5 / 2}}$

Here $\mu_{1}$ is the relative permeability of the current medium (for the polymers $\mu_{1}=1$ ), $d$ is the particle diameter, $l_{0}$ is the distance between the nearest particles centers in the unsheared chain, $\epsilon$ is the shear, $m=\pi d^{3} J_{p} / 6$ is magnetic moment of the particle, $J_{p}$ is the particle magnetization of saturation. Parameter $l_{0}$ in this model is considered as adjusted. The shear stress, provided by the interacting magnetic particles, can be determined as

$\sigma=\frac{\partial U}{\partial \epsilon}$

The shear modulus can be defined by using the standard relation $G=\frac{\partial \sigma}{\partial \epsilon}$. Besides $l_{0}$, this model includes the second adjusted parameter which relates to the reluctance of the sample.

Experimental study of effect of magnetic field on the shear modulus $G$ of the composites with the particles volume concentrations $\varphi=10,20$ and 30 per cent have demonstrated the relative change $(G(H)-G(0)) / G(0)$ of the modulus about 30,39 and 31 per cent respectively. By fitting the adjusted parameters, the model allows to achieve good agreement with the experiments presented in this work. 
In the simplest theoretical approaches the chains are presented as straight aggregates perfectly aligned along the field of polymerization. Obviously, this perfect structure in real situations can be achieved not always. A model of magnetic elastomers with fluctuations of the particles positions in the chains was suggested in [63].

It was shown that, depending on the details of the fluctuations, magnetic field, aligned along the chains, can induce either elongation or contraction of the sample in the field direction. Magnetic field, applied perpendicularly to the chains, induces elongation of the composite. The microscopic physical cause of that is the rearrangement of the particles in the chains under the field action. The developed model demonstrates important role of the chain fluctuations in the macroscopical deformation of the composites. The obtained theoretical results allowed to get quite good agreement with experiments, performed in [63], both for the magneto-striction and for the shear deformations of the system.

A theoretical model for calculation of the elongation stress in the magnetic elastomers with the chain-like structures in the absence of external magnetic field is suggested in [74]. This model includes analysis of the elastic effects in the thin gaps between the particles in the chains. The following formula is obtained for the macroscopical elastic stress:

$\sigma=\frac{E_{e f f} a^{2}+E_{m}\left[(a+L)^{2}-a^{2}\right]}{(a+L)^{2}} \varepsilon$

Here $\varepsilon$ is the macroscopical strain, $a$ is the particle radius,

$$
L=\sqrt{\frac{4 a^{2}}{3 \varphi\left(2+\frac{g}{a}\right)}}-a
$$

$\varphi$ is volume concentration of the particles, $g$ is thickness of gaps between poles of the nearest particles in the chains, $E_{m}$ is the Young's modulus of the matrix, $E_{\text {eff }}$ is some effective elastic modulus of the structure, determined by the deformation of the polymer in the gaps between the particles.

The analytical model of [74] has been verified by computer calculations and comparison of the results with experiments. The agreement was quite good. Authors have concluded that the structured magnetic elastomers can be described in the single-chain approximation, i.e. ignoring any interactions between the chains.

The problem of effect of the chain-like structures on the elastic properties of magnetic elastomers theoretically have been studied also in [75]. The elastomer was considered under the 
action of magnetic field, parallel to the chains. Authors concluded that the perfectly straight chains induced decrease of the tensile modulus while magnetic field increasing, whereas the wavy chains enhanced this modulus. Both straight and wavy chains, in this model, increased the composite shear modulus. The perfectly straight chains, in this model, provided the higher change of the modulus than the wavy ones.

To the best of our knowledge, in literature there is no another evidence on decrease of the ferrogels tensile modulus under the field action. Thus, the conclusion on the softness, under the field action, of the composite with the perfect chains, to our opinion, requires careful relation and further studying.

Possible, the first phenomenological consideration of magnetistriction of an elastic magnetizable (polarizable) sphere has been done in the classical book [53]. Analysis is based on the minimization of the total free energy of the sphere, placed in external magnetic (electrical) field, taking into account change both of the magnetic (electrical) and the elastic parts of the energy as a consequence of the sample small deformations. It is supposed that the deformation inside the sphere is uniform and the striction leads to transformation of the sphere to an ellipsoid of revolution. One needs to note that the assumption on the uniform transformation of the sphere to the ellipsoid has been used in [53] without justification.

In more details the problem on the sphere magnetostriction has been considered in [76] by using direct solution of equations of the Hook's deformation and magnetization of the sample with the corresponding boundary conditions on its surface. Like in [53], only small deformations of an incompressible sphere with the linear law of magnetization have been considered

In the cylindrical coordinate system, with the polar axis $O z$ aligned along the external field, the components of the $f$ displacement vector $\boldsymbol{u}$ in [76] have been presented as:

$u_{\rho}=A_{1} \rho+A_{2} \rho^{3}+A_{3} \rho z^{2}$

$u_{z}=C_{1} z+C_{2} z^{3}+C_{3} z \rho^{2}$

where $z$ and $\rho$ are the cylindrical coordinates parallel and perpendicular to the field respectively. The six constants $A_{i}, C_{i}$ have been determined from the standard boundary conditions on the surface of the sphere taking into account the elastic and Maxwell stresses. Unlike the assumption made in [53], the deformation (8) is not uniform and indicates nonellipsoidal transformation of the sphere.

According to results of [53], the component of the deformation tensor on the sphere pole is:

$\varepsilon_{z Z}=\frac{4 \pi}{15} \frac{(\chi H)^{2}}{G}$ 
Here $H$ is magnetic field inside the non deformed sample, $\chi$ is magnetic susceptibility of the sample material, $G$ is its modulus of the shear elasticity. Note that formula (9), as in [53], is presented in the Gaussian system of units.

The result of [76], in the same system, reads:

$\varepsilon_{z z}=\frac{20 \pi}{57} \frac{(\chi H)^{2}}{G}$

The deformation (10), determined in [76], is $30 \%$ more than that in (9), however mathematically the approach of [76] is significantly more cumbersome than the simple energetic method of [53].

It should be noted that the model [76] does not take into account the change of the ferrogels susceptibility $\chi$ due to the sample deformation. The alternation of $\chi$ takes place as a consequence of the particles mutual disposition in the composite, and, therefore, change of their mutual magnetization. Phenomenologically effect of $\chi$ alternation on the internal stress in magnetizable media and on the magnetostriction of the spherical sample has been taken into account in [53]. It was shown that the components of tensor of susceptibility of an initially isotropic medium in the linear approximation with respect to the small deformations can be presented as:

$\chi_{i k}=\chi_{0} \delta_{i k}+a_{1} \varepsilon_{i k}+a_{2} \varepsilon_{k k} \delta_{i k}$

Here $\chi_{0}$ is the susceptibility of the non deformed medium, $\varepsilon_{l m}$ are the components of the tensor of deformation, $a_{1}, a_{2}$ are material constants, which must be determined either experimentally or from a microscopical consideration, $\delta_{i k}$ is the Kronecker tensor. A problem of magnetostriction of a spherical sample with the susceptibility (11) has been considered in [53] under the assumption on the uniform deformation of the sample placed in the uniform external magnetic field $H_{0}$. The result (in the Gaussian system) reads:

$\varepsilon_{z Z}=\frac{2 \pi M_{0}^{2}}{3 G}\left(\frac{2}{5}+\frac{a_{1}}{\chi_{0}^{2}}\right)$

Here $M_{0}=H_{0} \frac{3 \chi_{0}}{3+\chi_{0}}$ is magnetization of the non deformed sample, $\chi_{0}$ is its susceptibility.

Magnetostriction of ferrogels with the changeable susceptibility $\chi$ has been studied in [77] on the basis of solution of the equations of the elastic Hook's deformation and linear magnetization of the sample. The change $\delta \chi$ of the susceptibility, due to the composite deformation, has been estimated as in (11); parameters $a_{1}, a_{2}, a_{3}$ were used in form calculated in [78]. 
In the framework of approach of [78], the macroscopical deformation of the sample leads to change $\delta g$ of the of the pair function $g$ of the particles spatial distribution. Mathematically the change $\delta \chi$ of the composite suscpeptibility is determined through the function. The last function has been determined in [78] in the framework of the following approximations. The first one is the assumption that the distribution function $g_{0}$ in the non deformed sample is determined from the relation:

$g_{0}=\begin{aligned} & 0, \text { when } l<d \\ & 1, \text { when } l>d\end{aligned}$

Here $l$ is the distance between the particle centers, $d$ is the particle diameter. As well known, this approximation is valid only for low concentrated isotropic systems, i.e. for the ideal gas of the particles. The second approximation is in assumption on the affine deformation of the sample, i.e. that the vector of mutual displacement of two particles has the same mathematical form as the vector $\boldsymbol{u}$ of the sample macroscopical deformation. This affine approximation neglects the local character of the matrix deformation near the particles and the elastic effects which take place because of impossibility of the particles interpenetration. That is why the affine approximation can lead only to qualitative results.

In the framework of these approximations, in [78], the parameters $a_{1}$ and $a_{2}$ were determined as:

$a_{1}=-\frac{2}{5} \chi_{0}^{2}$

$a_{2}=-\frac{1}{3} \chi_{0}\left(\chi_{0}+3\right)+\frac{2}{15} \chi_{0}^{2}$

The negative sign of $a_{1}$ indicates that the susceptibility of the sample decreases while the sample elongation.

Substituting $a_{1}$ from (13) into (12), one can conclude that the spherical sample mustn't be deformed $\left(\varepsilon_{z z}=0\right)$. This result is a consequence of the assumption [53] on the uniform deformation of the sample and the estimate (13) of the parameter $a_{1}$.

In contrast, calculations of [77], based on the direct solution of the boundary problem for the sphere deformation, give:

$\varepsilon_{z Z}=\frac{8 \pi}{95} \frac{M_{0}^{2}}{G}$ 
The relation (13) for $a_{1}$ have been used here; the result is presented in the Gaussian system of units.

For the ellipsoid of revolution with the arbitrary ratio $r=a / b$ of the axis $a$ of the ellipsoid symmetry to its diameter $b$, the following formula has been suggested in [77] as a generalization of (14):

$\varepsilon_{z Z}=\frac{4 \pi}{15} \frac{M_{0}^{2}}{G}\left[\frac{175 r^{2}\left(7+10 r^{2}+8 r^{4}\right)}{\left(3+24 r^{2}+8 r^{4}\right)\left(23+24 r^{2}+48 r^{4}\right)}-1\right]$

Here, again, $M_{0}$ is magnetization of non deformed sample $\left(M_{0}=\frac{\chi_{0}}{1+N(r) \chi_{0}} H_{0}, N(r)\right.$ is the ellipsoid demagnetizing factor). Magnetic field is parallel to the axis $a_{\text {. }}$ Unfortunately, derivation of (15) is not given in [77].

The deformation $\varepsilon_{z z}$ is negative, i.e. the sample contracts in the field direction, when it is either significantly oblate $\left(r<r_{1} \approx 0.32\right)$ or prolate $\left(r>r_{2}>1.35\right)$. The deformation is positive (the sample elongates) for the intermediate magnitudes of $r$. This results was named in [77] as the "Procrustes effect".

Qualitatively the same conclusion has been made in [79] on the basis of the energetic approach under assumption on the uniform deformation of the sample. The threshold values of the aspect ratio $r_{1,2}$, corresponding to the change of the sign of $\varepsilon_{z z}$ (change type of deformation) in [79] are a little different from those, determined by the formula (15). It should be noted, that the magnitude of the parameter $a_{1}$, obtained in [79], differs from (13), determined in [78] and used in [77], in spite of the fact that the same approximations of the affine deformation of the sample and random, like in ideal gas, distribution of the particles in the matrix has been used in [79].

In the framework of this approach, effect of the internal chains on the type of the sample magnetostriction was studied in [79] under the assumption that the chains are perfectly aligned along the axis of the sample symmetry; the deforming field has the same direction as the chains. The results show that, if the gaps between the nearest particles in the chains are compressible, the reduction of the gap thickness, under the field action, leads to the macroscopical contractionof the sample. This conclusion is in 
agreement with the results of experiments and modeling of $[47,63]$. If the gaps are incompressible, either the gap thickness is negligible, magnetic interaction between the chains induces elongation of the sample.

According to the energetic approach $[53,79]$ the change of the magnetic free energy of a magentizable ellipsoid, after the sample small elongation (contraction), can be presented as

$$
\delta F_{m}=-\frac{1}{8 \pi} \frac{H_{0}^{2} V}{(1+\chi N)^{2}}\left[\delta \chi-\chi^{2} \delta N\right]
$$

Here $H_{0}$, again, is the field, external with respect to the sample, $V$ and $N$ are its volume and demagnetizing factor respectively, $\chi$ and $\delta \chi$ are the sample susceptibility and its change in the field direction. The physical deformation of the composite leads to decrease of its free energy, i.e. it must correspond to the inequality $\delta F_{m}<0$. The relation (16) shows that the type (elongation or contraction) of the sample deformation is a result of competition between two following factors. The first one is the effect of the sample demagnetizing field. Since $\delta N<0$ at any sample elongation, the demagnetizing mechanism always stimulates elongation of the sample. On the other hand, the change $\delta \chi$ of the susceptibility at the sample elongation can be either negative, or positive. In the first case this factor stimulates contraction; in the second case it induces elongation of the sample. Calculations of $[77,79]$ show that in the composites with the random (ideal gas) distribution of the particles the inequality $\delta \chi<$ 0 is held. Effect of change of the susceptibility dominates over the shape effect of the demagnetizing field when the aspect ratio $r$ is small or large enough. Analysis of [79] demonstrates also that magnetic interaction between the incompressible chains leads to positive change of the susceptibility $(\delta \chi>0)$ at the sample elongation. Therefore, both factors, the demagnetizing field and the change of the composite susceptibility lead to elongation of the composite with the chains.

In disagreement with theoretical prediction $[77,79]$ of the Procrustus effect, experiments [52], with the samples containing chaotically distributed magnetizable 
particles of $30 \%$ volume concentration, have demonstrated only elongation of the ferrogels in the wide range of the shape factor $r$, including the values of $r$, for which the models $[77,79]$ predict the sample contraction.

In order to understand the physical cause of the disagreement between experiments [52] and the models [77,79], the problem of magnetostriction of a magnetic composite with the randomly distributed particles has been considered in [80]. Unlike the models $[77,79]$, non affine deformation of the sample, on the microscopical level, has been taken into account; the function $g_{0}$ of the mutual distribution of the particles has been used in form of the pair function in a gas of hard spheres, taking into account the short-ranged correlations between the particles [81]. The following result has been obtained for the change of the composite susceptibility

$$
\delta \chi=-\frac{18}{5}(0.2-1.234 \varphi) \varphi^{2} \varepsilon
$$

where $\varepsilon$ is the relative elongation in the field direction, $\varphi$ is volume concentration of the particles. For positive $\varepsilon$ (i.e for the sample elongation) the inequality $\delta \chi<0$ is held when $\varphi<$ 0.162 and the opposite inequality $\delta \chi>0$ takes place when $\varphi>0.162$. This conclusion is in agreement with the results of models [77,79] that elongation of the composites with the ideal-gas distribution of the particles leads to decrease of their effective susceptibility. In its turn, this induces the Procrustus effect - for small concentrations of the particles the ellipsoid shrinks in the field direction when the aspect ratio either $r$ is less than some threshold $r_{1}(\varphi)$ or more than another one $r_{2}(\varphi)$. Unlike the models [77,79], calculations of [80] show that $r_{1}$ and $r_{2}$ depend on the particle concentration $\varphi$.

When $\varphi>0.162$, the interparticle correlations lead to increase of the susceptibility $(\delta \chi>$ 0 ) while the sample elongation. In this case, both factors - change of the sample demagnetizing factor and change of its susceptibility induce elongation of the composite in the field direction. Taking into account that in experiments [52] the volume concentration $\varphi$ of the particles was $30 \%$, this result explains the noted contradiction between the theories [77,79] and experiments [52] - the results [77,79] are applicable only for very dilute suspensions with small concentrations $\varphi$.

The conclusion on the effect of the interparticle correlations on the type of magnetostriction is supported by the computer simulations of [82]. Two-dimensional model of a square shaped 
ferrogel has been considered. The spatial disposition of the magnetizable particles was supposed isotropic and chaotic with some correlations because of the steric interaction between them. External field has been applied in the plane of the square. The simulations have demonstrated elongation of the "sample" in the field direction. However, when the correlated particles were considered as non magnetic, only the non correlated ones were magnetizable, the model contracted, similar to the predictions of $[77,79]$.

Experimentally magnetodeformation of a ferrogel ball with randomly distributed particles of a magnetic filler has been studied in [83]. Practically incompressible, very soft gel, softened under the load, was used as the composite matrix. During measurements (1000sec) the shear modulus of the loaded matrix decreased, approximately, from 1300 to $400 \mathrm{~Pa}$. The weight concentration of the magnetite nanoparticles in the ferrogels was about $24.5 \%$. The ferrogels ball was placed in uniform magnetic field ranged from 0 to $30 \mathrm{kA} / \mathrm{m}$. Small elongation of the sample in the field direction has been detected. The measurements demonstrated that the elongation was about $70 \%$ more than that calculated from the model [76]. It should be noted that the model [76] predicts the maximal magnetodeformation as compared with the results $[53,77,79]$. Possible, the relatively strong magnetomechanical effect can be explained by formation of the chain-like aggregates. Indeed, analysis of $[79,84]$ showed that formation of the chains in the system of ferromagnetic particles induces and enhances the sample elongation. Unfortunately, there is no information on the size distribution of the particles, used in experiments [83]; this does not allow making quantitative analysis of the observed effect.

In majority of theoretical works the energy $U_{m}$ of magnetic interaction between the particles has been estimated by using the simplest dipole-dipole approximation. For spherical ferromagnetic particles with permanent magnetic moments this approach is exact, but it is not so for the magnetizable particles. Possible, the most mathematically rigorous method for calculation of the energy $U_{m}$ of interaction of two linearly magnetizable particles has been developed in [85]. However this approach leads to cumbersome results, which practical usage is not quite convenient.

The following extrapolation formula

$U_{m}(\gamma, q)=-3 \mu_{0} \mu H_{0}^{2} v \sum_{k=3}^{7}\left(\frac{\alpha-1}{\alpha+2}\right)^{p_{k}}\left[\frac{a_{k}}{\left(q-b_{k}\right)^{k}}+\frac{c_{k}}{\left(q-d_{k}\right)^{k}} \cos ^{2} \gamma\right]$

for this energy $U_{m}$ has been suggested in [86]. Here $\mu_{0}$ again is the vacuum permeability, 
$\alpha=\mu_{p} / \mu, \mu_{p}$ and $\mu$ are the permeability's of the particles and matrix respectively, $q=l / a, l$ is radius vector, connecting centers of two interacting particles, $\gamma$ is the angle between $\boldsymbol{l}$ and the applied field $\mathbf{H}_{0}, v$ is volume of the particle. The linear low of the particles magnetization is supposed in (17).

Parameters $a_{k}, c_{k}, d_{k}$ and $p_{k}$ are tabulated in [86]. The calculations show that the force of attraction of two closely situated particles at $\gamma=0$ (pole to pole position) is an order of magnitude more than that calculated in the dipole-dipole approximation. The force of repulsion at $\gamma=\pi / 2$ is slightly less than that is the dipole-dipole approach. As well known, in the last approximation, the angle $\gamma_{c}$, corresponding to transition from the aria of attraction to the aria of repulsion between the particles, satisfies to the equality $\cos \gamma_{c}=1 / \sqrt{3}$, i.e. $\gamma_{c} \approx 54^{\circ}$. The formula (17) demonstrates that the angle $\gamma_{c}$ depends on the dimensionless distance $q$ between the particles, and at $q=2$, i.e. when particles are in contact, $\gamma_{c} \approx 84.3^{\circ}$. This means that the region of attraction between magnetic particles is significantly more than the dipole-dipole approximation predicts. This conclusion is important for theoretical study not only of the ferrogels with internal structures, but also for analysis of rheological effects in liquid magnetic suspensions.

Equation (17) leads to significant deviations from the results of the dipole-dipole approximation only when the particles are very close and $2<q<3$. When $q>3$, the difference between (17) and the predictions of dipole-dipole approach is insignificant.

In real magnetic polymers the approximation of linear magnetization of the particles is valid only in the case of weak magnetic fields. Analysis of energy of interaction of the non-linearly magnetizable particles has been done in [87]. In this approach each of the interacting particles has been considered as situated in an uniform magnetic field, which is the sum of the applied field $\mathbf{H}_{0}$ and the field $\mathbf{H}_{\mathrm{d}}$, created by the other particles. The energy of the interparticle interaction was estimated in the dipole-dipole approximation; magnetic moment $\boldsymbol{m}$ of the particle was determined by using the self-consistent method, under assumption that the particle was situated in the field $\mathbf{H}_{0}+\mathbf{H}_{\mathrm{d}}$ and the field $\mathbf{H}_{\mathrm{d}}$ was created by the second particle with the same moment $\boldsymbol{m}$. Magnetization of the particle was assumed homogeneous inside its body and estimated by using the non-linear Frolich-Kennelly relation. Numerical calculations have been performed for verification of this model. The analytical approach is in good agreement with the computer results when the applied field $\mathrm{H}_{0}$ is more than magnetization of saturation $\mathrm{M}_{\mathrm{s}}$ of the particle material. 
Another approach, which takes into account non uniform distribution of magnetization inside the particles, has been suggested in $[88,89]$. Two closely situated particles placed in a homogeneous magnetic field have been considered. Near poles of the particles some zone with saturated magnetization was supposed. The radius of this zone was estimated as function of applied field $\mathrm{H}_{0}$ and the thickness of the gap between the particles. Outside the saturated zone the field in the gap was assumed the same as the field created by linearly magnetizable particles. The energy and force of magnetic interaction between the particles was estimated. The approach $[88,89]$ is especially useful for analysis of the systems of highly permeable particles.

Computer simulations of magnetic gels deal with two opposite limiting models. The first one considers the composites as systems of polymer chains cross-linked by magnetic particles. Physically this model corresponds to magnetic hydrogels, which have been presented in the experimental works [90-92]. A brief overview of works on these materials can be found in [93].

The computer models of the magnetically cross-linked hydrogels have been developed in $[94,95]$. Schematically these systems are illustrated in Fig.8

It was assumed in $[94,95]$ that the magnetic particles had permanent magnetic moments; elastic properties of the polymer chains have been modeled by the Hook's springs.

A two-dimensional computer model of the hydrogels has been developed in [94]. The size of the particles was supposed typical for modern ferrofluids, i.e. about $10 \mathrm{~nm}$. It was noted that apart from a qualitative description of three-dimensional systems, such $2 D$ model can be used to study the properties and behavior of very thin films. The simulations were performed by using the method of molecular dynamics. In the simulations a constant external force, aligned along external magnetic field, was applied to the first and last beads of each polymer chain. Both the longitudinal and lateral deformations of the polymer network were measured. The computer experiments have demonstrated auxestic properties of the model (the Poison coefficient has appeared negative). One needs to note that, to the best of our knowledge, the auxetic effects have not been detected in experiments with the magnetic polymers.

The paper [95] also deals with the study of deformation, microstructure, and magnetic properties of the two dimensional gels by using the molecular dynamic simulation. Two models of the gel are considered there. In the first one, super paramagnetic particles with the Neel mechanism of remagnetization are situated directly in the polymer chains. In the beginning of simulation, the moments are orientated randomly. Under the action of the applied field, 
magnetic moments align in the field directions, particles attract in the field direction and repulse in the perpendicular one. The simulations demonstrate shrink of the sample in the direction perpendicular to the applied field and elongation in the field direction. Physically this result can be explained by reorientation of the gel chains, containing magnetic particles. Because of magnetic interaction between the particles, this chains tend to align along the field.

In the second model, considered in [95], magnetic particles are situated in the nods of the gel net. In the other words, the particles serve as cross-linkers of the network. The nod particles are connected by chains which are attached to specific spots on the particles' surface. This type of bonding makes the particle rotation coupled to the polymer matrix. Unlike the first model, this rotation cannot occur freely and involves the chains attached to the surface. The dipole-dipole interaction between the particles is not taken into account in this model. Under the action of the field, the sample, in this model, shrinks isotropically, as the chains get rolled up equally in all directions around the rotating magnetic particles.

Thermodynamic functions (internal and free energies), of the cross-linked hydrogels have been calculated in computer simulations [96].

Ferrogel as an elastic continuum with embedded identical magnetic spheres was considered in computer simulations of [97]. The particles were considered as ferromagnetic with permanent magnetic moments. Volume concentration of the particles was varied in the range 6-11\%. The Monte Carlo simulations were performed to generate typical microstructures and to determine the responses of the resulting ferrogel to applied magnetic fields. It was supposed that the dipole moment was free to rotate unhindered by interactions between the gel matrix and the magnetic grain. Magnetostriction, elastic modules and magnetization of the composites have been studied in [97].

Three situations have been considered. In the first one, the gel was treated as prepared without magnetic field. Therefore initially the particles distribution and orientation of their magnetic moments was random and isotropic. The samples of the second and third kinds were polymerized under the action of a permanent magnetic field. In the second set of simulations the samples were placed in a field with the same direction as the field of polymerization. The third sets have been performed under the assumption that the acing field was perpendicular to the field of polymerization.

In all cases the samples contracted in direction of the acting field. It should be noted that the conclusion on the contraction, in the field direction, of the samples with chaotic disposition of the particles, disagrees with the experiments of [52,57] where only elongation of the sample have been observed. Possibly, the contraction [97] takes place since effects of the demagnetizing field have not been taken into account in these simulations. At the same time, the low concentrated 
systems have been studied in [97], whereas in [52,57] the composite with significantly higher concentrations have been used. Thus, effect of correlations between the particles positions, which stimulates elongation of the sample [80], was weak in simulations [97] and significant in experiments [52,57].

The conclusion of [97] on the contraction of the gel under the action of the field, perpendicular to the field of polymerization, disagrees with the observed elongation of the samples in the experiments [63] and is worse further studying.

In the cases of the isotropic medium and composite with the axis of anisotropy, parallel to the acting field, simulations [97] demonstrate increase with the appplied field of the sample Young's modulus, corresponding to deformations in directions parallel and perpendicular to the field. The modules of the systems with the internal structure were more than those of the isotropic systems. In the case of the field, perpendicular to the internal structures, the Young's modulus, determined in the direction of the acting field, increased with the field, whereas the modulus, corresponding to the perpendicular directions, decreased with the field.

\section{CONCLUSION}

Magnetopolymer composites (ferrogels, magnetic hydrogels, magnetic elastomers) present new kind of smart soft materials. Combination of the properties of polymer materials with high response on magnetic field is very perspective for industrial and medical application, as the magnetically controlled dampers and shock absorbers, sensors, artificial muscles and other biological tissues, the scalfolds for growing of the cell cultures. Macroscopical physical and mechanical properties of these systems, futures of their behavior and reaction on the applied magnetic field are very sensitive to the properties of the polymer matrix, size and law of spatial distribution of the particles. Fundamental physical causes of many observed phenomena are still unclear. Some experimental, computer and theoretical results contradict to each other. That is why the study and synthesis of these composites presents very perspective and challenging problem.

Acknowledgments. This work has been supported by the Russian Scientific Foundation, project 14-19-00989. Financial support by project FIS2013-41821-R, MINECO, Spain, is also acknowledged.

1. G. Filipcsei, I. Csetneki, A. Szilagyi and M. Zrınyi, Adv. Polym. Sci., 206, 137 (2007)

2. A. Boczkowska and S.F. Awietjan, Materials Science Forum 636-637, 766 (2010)

3. S.Dyke, B.Spencer and M.Sain, J.Carlson, Smart Materials and Structures, 5, 565 (1996) 
4. A.Occhiuzzi, M. Spizzuoco and G.Serino, Smart Materials and Structures, 12,703 (2003)

5. F.Carmona and C.Mouney, J Mater. Science, 27, 5, (1992)

6. J.F.Feller, I.Linossier and Y.Grohens, Mat. Letters, 5764 (2002)

7. B.Lundberg and B.Sundqvist J. Appl. Physics, 60, 1074 (1986)

8. S.Jin, J.Mottine, J.John and R.Sherwood, U.S. Patent. 4,644,101(1987)

9. J.Barkauskas, Talanta, 441107 (1997)

10. Y.S.Kim, S.C.Ha, Y.Yang, Y.J. Kim, S.M.,Cho S M, H.Yang and Y.T.Kim, Sens. Actuators. $B, 108,285$ (2005)

11. S.K.Bhattacharya Metal Filled Polymers (New York: Marcel-Dekker) (1992)

12. J.A.Fulton, R.C. Moore, W.R.Lambert and J.J.Mottine Proc. $39^{\text {th }}$ Electronic Components, Conf. IEEE 39, 71 (1989)

13. M. van Bruggen and J. van Zon, Sens. Actuators A Phys., 158, 240 (2010)

14. S.van Berkum, J.T. Dee, Albert P. Philipse and B.H. Erné, Int. J. Mol. Sci., 14, 10162 (2013)

15. M.Bañobre-López, Y.Piñeiro-Redondo, R. de Santis, A.Gloria, L.Ambrosio, A.Tampieri, et al. J Appl Phys., 109, 07B313 (2011)

16. N.Bock, A.Riminucci, C.Dionigi, A.Russo, A.Tampieri, E.Landi, et al. Acta Biomater. 6, 786 (2010)

17. B.Das, M.Mandal, A.Upadhyay, P.Chattopadhyay, N. Karak., Biomed. Mater., , 8, 035003 (2013)

18. R.de Santis, A.Gloria, T.Russo, U.d'Amora, S.Zeppetelli and C.Dionigi, J.Appl. Polym. Sci., 122,3599 (2011)

19. A.Gloria, R.Russo, U. d'Amora, S. Zeppetelli, T.d'Alessandro, M. Sandri, et al., J. R. Soc Interface, , 10, 20120833, (2013)

20. S.H.Hu, T.Y. Liu, C.H.Tsai, S.Y.Chen, J Magn Magn Mater., 310, 2871 (2007)

21. H.Hu, W. Jiang, F.Lan, X. Zeng, S.Ma, Y.Wu, et al, $R S C, A d v ., 3,879$ (2013)

22. K.Lai, W.Jiang, J.Z.Tang, Y.Wu, B.He, G.Wang, et al., RSC Adv. 2, 13007 (2012)

23. Y.Li, G.Huang, X.Zhang, B.Li, Y.Chen, T.Lu, et al, Adv Funct Mater. 23, 660 (2013)

24. S.Panseri, C.Cunha, T.'Alessandro, M.Sandri, G.Giavaresi, M.Marcacci, et al., J Nanobiotechnology, 10, 32 (2012)

25. H.Skaat, O.Ziv-Polat, A.Shahar, D.Last, Y.Mardor, S.Margel., Adv Healthc Mater.; 1, 168 (2012)

26. A.Tampieri, E.Landi, F.Valentini, M.Sandri, T.d'Alessandro, V.Dediu, et al., Nanotechnology, 22, 015104 (2011)

27. A.Tampieri, T.d'Alessandro, M.Sandri, S.Sprio, E.Landi, L.Bertinetti, et al., Acta Biomater. $8,843(2012)$ 
28. X.B. Zeng, H.Hu, L.Q.Xie, F.Lan, W.Jiang, Y.Wu, et al., Int J Nanomed., 7, 3365 (2012)

29. X.B. Zeng, H.Hu, L.Q.Xie, F.Lan, Y. Wu, Z.W.Gu. J Inorg Mater., 28, 79 (2013)

30. Y.Zhu, F.Shang, B.Li, Y.Dong, Y.Liu, M.R.Lohe, et al., J Mater Chem B., 1, 1279 (2013)

31. O.Ziv-Polat, H.Skaat, A.Shahar, S.Margel., Int J Nanomed, 7, 1259 (2012)

32. R.K.Singh, K.D.Patel, J.H. Lee, E.J.Lee, J.H.Kim, T.H.Kim, et al., PLOS ONE, , 9, e91584 (2014)

33. M. T. Lopez-Lopez, G.Scionti, A.C. Oliveira, J.D. G. Duran, A. Campos, M. Alaminos, I.A.Rodriges, PLOS ONE, DOI:10.1371/journal.pone.0133878 July 24, (2015)

34. M. Zrinyi, L. Barsi,A. Buki, J. Chem. Phys., 104, 8750 (1996)

35. J. A. Galicia, F. Cousin, E. Dubois, O. Sandre, V. Cabuil and R. Perzynski, Soft Matter , 5, 2614 (2009)

36. R. Langer, J.P.Vacanti , Science, 260, 920 (1993).

37. J.A.Baddour et al. Birth Defects Res. Part C-Embryo Today-Rev. 96, 1 (2012).

38. Bañobre-Lopez M, Y. Piñeiro-Redondo, R. De Santis, A. Gloria, L. Ambrosio, A.

Tampieri, V. Dediu and J. Rivas, J. Appl. Phys. 109, 07B313 (2011).

39. S.Panseri, A.Russo, C.Cunha, A.Bondi, A.Di martini, S.Patella and E.Kon, Knee Surg. Sports Traumatol. Arthrosc. 20, 1182 (2012).

40. S.Panseri, C.Cunha, T.D'Alessandro, M. Sandri, A. Russo, G.Giavaresi, M.Marcacci, C.T. Hung, A. Tampieri, PLoS One, 7, e38710 (2012).

41. L. V. Nikitin, L. S. Mironova, G. V. Stepanov and A. N. Samus, J.Polym. Sci. A, , 43, 443 (2001)

42. G. V. Stepanov, S. S. Abramchuk, D. A. Grishin, L. V. Nikitin, E. Y. Kramarenko and A. R. Khokhlov, Polymer, 48, 488 (2007)

43. G. Y. Zhou and Z. Y. Jiang, Smart Mater. Struct., 13, 309 (2004)

44. Y. L. Raikher and O. V. Stolbov, J. Appl. Mech. Tech. Phys., , 46, 434 (2005)

45. G. Y.Zhou and J. R. Li, Smart Mater. Struct. 12, 859 (2003)

46. Y. Shen, M. F. Golnaraghi and G. R. Heppler, J.Intell. Mater. Struct, ,15,27 (2004)

47. E.Coquelle and G.Bossis, J.Advanced Science, 17, 132, (2005)

48. M. Zrınyi, L. Barsi and A. Buki, J. Chem. Phys. 1048750 (1996)

49. M. Zrınyi, L. Barsi and A. Buki, Polym. Gels Networks 5415 (1997)

50. M. Zríny, L. Barsi, D. Szabó and H.-G. Kilian, J. Chem. Phys. 106, 5685 (1997)

51. D. Szabo, G. Szeghy, and M. Zrınyi, 31, 6541 (1998)

52. G. Diguet, E. Beaugnon and J.Y. Cavaille, J. Magnetism and Magnetic Materials, 322, 3337 (2010) 
53. L.D. Landau, E.M. Lifshitz, Electrodynamics of Continuous Media, Pergamon Press, Oxford, (1960)

54. N. Kchit, P. Lancon and G. Bossis, J.Phys.D.Appl.Phys.42, 105505 (2009).

55. N. Kchit, P. Lancon and G. Bossis, J.Phys.D.Appl.Phys.42 105506 (2009)

56. C.Bellan and G.Bossis, Int. Journal of Modern Physics B, , 16, 2447 (2002)

57. G.Stepanov, D.Borin, Yu.Raikher, P.Melenev and N.S. Perov, J. Phys.: Condens. Matter, 20 204121 (2008)

58. Z.Varga, G.Filipsei and M.Zrini, Polymer, , 47, 227 (2006)

59. Bica, E.M. Anitas L.M.E. Averis, J. Industrial and Engineering Chemistry 2886 (2015)

60. G. Schubert and P.Harrison, Polymer Testing 42122 (2015)

61. Y. Shen, M. F. Golnarach and G. R. Heppler, J. Intellegent Material Systemsand Structures, $15,27(\mathbf{2 0 0 4 )}$

62. V. Rao, S Maniprakash, S. M. Srinivasan and A. R. Srinivasan, Smart Mater. Struct. 19 085019 (2010)

63. K. Danas, S.V.Kankanala and N.Triantafyllidis, J. Mechanics and Physics of Solids, 60120 (2012)

64. P.Bender n, A.Tschöpe and R.Birringer, J.Magnetism and Magnetic Materials, 346, 152, (2013)

65. T. Mitsumata, T.Wakabayashi, T. Okazaki, J. Phys. Chem. B 112, 14132 (2008)

66. T. Mitsumata, S. Ohori, N. Chiba and M. Kawai, Soft Matter, 9, 10108 (2013)

67. K. Nagashima, S. Kanauchi, M. Kawai, T.Mitsumata, S.Tamesue and T. Yamauchi, J. Appl. Physics, 118, 024903 (2015)

68. T. Mitsumata and S.Ohori, Polym. Chem., 2, 1063 (2011)

69. J. Wu, X. Gong, Y. Fan, H. Xia, Soft Matter, 7, 6205 (2011)

70. R.M.Christensen, Mechanics of Composite Materials, Krieger Publishing Company, 1991

71. D.Borin, D.Gunter, C. Hintze, G Heinrich and S.Odenbach, J. Magnetism and Magnetic Materials 324 3452-3454 (2012)

72. L. C. Davis, J.Appl.Physics, 85, 3348 (1999)

73. M.R. Jolly, J. D. Carlson, B. C. Muñoz, T.A. Bullions, Journal of Intelligent Material Systems and Structures, 6, 613-622 (1996)

74. E. Coquelle, G. Bossis, D. Szabo and F. Giulieri, Journal of Materials Science, 41(18):5941(2006)

75. Y. Han, W. Hong, L.Ann and E. Faidley, International Journal of Solids and Structures 50 $2281(\mathbf{2 0 1 3})$ 
76. Yu.L. Raikher and O.V. Stolbov, Journal of Magnetism and Magnetic Materials 258-259, 477 (2003)

77. K. Morozov, M.Shliomis and H.Yamaguchi, Physical Review E., 79, 040801 (2009)

78. Y. M. Shkel and D. J. Klingenberg, J. Appl. Phys. 83, 7834 (1998).

79. A. Zubarev, Physica A 392, 482 (2013)

80. A.Yu. Zubarev, D.Yu. Borin, J. Magnetism and Magnetic Materials, 377, 373 (2015)

81. R.Balesku, Equilibrium and non equilibrium statistical mechanics, V.1. Wiley-Interscience publication. John Wiley \& Sons, (1975).

82. O.V.Stolbov.,Y.L Raikher and M. Balasoni, Soft Matter, , 7, 8484 (2011)

83. C. Gollwitzer, A.Turanov, M.Krekhova, G.Lattermann, J.Chemical Physics, , 128, 164709 (2008)

84. A. Zubarev, Soft Matter, 9 (20), 4985 (2013)

85. D.J. Jeffrey, Proc.Royal Soc. London A, 335, 355A (1973).

86. M. Biller, O. V. Stolbov and Yu. L. Raikher, J.Applied Physics, 116, 114904 (2014)

87. A. M. Biller, O. V. Stolbov andYu. L. Raikher, Physical Review E., , 92, 023202 (2015)

88. J. M. Ginder and L. C. Davis, Appl. Phys. Letter, 65, 3410 (1994)

89. J.M.Ginder, L.C.Davis, L.D.Elie, Int. J. Modern Physics B., 10, 3293 (1996).

90. M. Bonini, S. Lenz, R. Giorgi and P. Baglioni, Langmuir, 23, 8681 (2007)

91. R. Messing, N. Frickel, L. Belkoura, R. Strey, H. Rahn, S. Odenbach and A. M. Schmidt, Macromolecules, 44, 2990,(2011)

92. R. Barbucci, D. Pasqui, G. Giani, M. D. Cagna, M. Fini, R. Giardino and A. Atrei, Soft Matter, 7, 5558, (2011)

93. P. Ilg, Soft Matter, 9, 3465 (2013)

94. M. R. Dudek, B. Grabiec and K. W. Wojciechowski, Rev.Adv.Mat.Science, 14,167, (2007)

95. R. Weeber, S.Kantorovich and C.Holm, Soft Matter, 8, 9923 (2012)

96. G.Pessot, R. Weeber, C. Holm, H. Löwen and A.Menzel, J. Phys.: Condens. Matter 27, 325105 (2015)

97. D. S. Wood and P. J. Camp, Physical Review E., 83, 011402 (2011) 